\title{
Observational studies
}

Ann-Christin Sannes*, Jan Olav Christensen, Dagfinn Matre, Morten Birkeland Nielsen and Johannes Gjerstad

\section{Patterns of pain complaints and insomnia symptoms are associated with abusive supervision in the Norwegian working population: a latent class analysis}

https://doi.org/10.1515/sjpain-2021-0124

Received July 8, 2021; accepted October 5, 2021; published online October 25, 2021

\begin{abstract}
Objectives: Previous findings suggest that abusive supervision, i.e., subordinates' perceptions of their supervisor's behaviours as hostile (excluding physical aggression), may increase the risk of health complaints. In addition, recent data suggest that the FKBP5 genotype rs9470080 important in the regulation of cortisol release, influences the same outcome. Adding to this complexity, different health complaints often co-occur. The present study aimed to (1) uncover patterns of pain complaints and insomnia symptoms by using latent class analysis, (2) determine whether abusive supervision or FKBP5 rs9470080 was associated with these patterns, and (3) examine the interaction between abusive supervision and FKBP5 genotype regarding pain and insomnia symptoms.

Methods: The data was collected through a national probability survey of 5,000 employees drawn from the National Central Employee Register by Statistics Norway. Abusive supervision was measured by a 5 -item version of the Tepper's 2000 scale. Pain and insomnia symptoms were measured by 5 items reflecting pain and 3 items reflecting insomnia. The FKBP5 rs9470080 genotyping was carried out using TaqMan assay.

Results: A total of 1,226 participants returned the questionnaire and the saliva kit sample. Based on these the latent class analyses revealed four classes based on response patterns of pain and insomnia symptoms. In the
\end{abstract}

\footnotetext{
*Corresponding author: Ann-Christin Sannes, National Institute of Occupational Health, Post address, Pb 5330 Majorstuen, 0304 Oslo, Norway, E-mail: ann-christin.sannes@stami.no

Jan Olav Christensen, Dagfinn Matre, Morten Birkeland Nielsen and Johannes Gjerstad, National Institute of Occupational Health, Oslo, Norway. https://orcid.org/0000-0003-0042-6389 (D. Matre)
}

regression analysis, abusive supervision was a significant predictor for the response patterns. However, neither the FKBP5 nor the interaction between abusive supervision and FKBP5 showed significant contributions.

Conclusions: In conclusion, awareness of the association between abusive supervision and the revealed four painand insomnia subgroups, and what separates them, may be important for prognosis and an optimal follow-up for those affected.

Keywords: genotype; latent class analysis; leadership; pain; sleep.

\section{Introduction}

Exposure to social stressors at work has previously been shown to increase the risk of several health complaints [1]. One such stressor is abusive supervision, i.e., subordinates' perceptions of his/her supervisor's hostile behaviours, excluding physical aggression [2]. Estimates suggest that $10-16 \%$ of workers are subjected to abusive leadership behaviours [3]. Exposure to hostile behaviours may lead to activation of the hypothalamic-pituitary-adrenal (HPA) axis [4-7]. The resulting cortisol release may also influence on the mechanisms underlying pain hypersensitivity and sleep problems [8]. A dysregulation of the HPA axis and its regulatory processes, is therefore important for many subjective health complaints.

One important regulator of the cortisol release is the FK506 binding protein 5 (FKBP5) expressed in the hypothalamus and pituitary gland [9]. In these structures, the FKBP5 protein affects ligand binding, intra-transcriptional processes, and receptor activation [10]. Earlier data suggest that the FKBP5 may be important in the negative feedback mechanism during stress; i.e., it regulates the glucocorticoid receptor (GR) sensitivity to cortisol [11, 12]. FKBP5 is also expressed in the thalamus, hippocampus, amygdala 
and pons [9]. These areas exhibit complex and interconnected activity during pain processing $[13,14]$ and sleep [15]. Recent data also show that the FKBP5 genotype, i.e. two copies of the rs $9470080 \mathrm{C}$ allele, is associated with both pain complaints and sleep problems [16].

Among adults in Norway up to $80 \%$ experience pain originating from the musculoskeletal system [17], most commonly low back pain [18] and neck pain [19]. Additionally, the prevalence of different types of headaches is high. Most common is tension-type headache $(60 \%$ among European citizens [20] and about 25\% globally [21]) and migraine (15\%) [20], which often co-occur [22], followed by cervicogenic headache $(17-50 \%)$ [23, 24]. The experience of pain is also influenced by other factors, such as sleep quality and/or quantity $[25,26]$. Earlier data show that sleep restriction may lead to many central neuronal consequences [27, 28], including regulation of neuroendocrine systems [29]. Due to the potential effects of such changes, sleep problems are therefore likely to be interrelated with somatic complaints including pain [25, 30]. A common term for problems relating to sleep is insomnia. Three important presentations of this problem are trouble falling asleep, trouble with coherent sleep and/or early awaking [31, 32].

To our knowledge, this is the first study exploring latent classes of pain complaints and insomnia by Latent Class Analysis (LCA) in the general Norwegian working population. LCA can be used to develop classifications based on both within-group similarities and betweengroup differences. The purpose of this study was (1) to uncover patterns of pain complaints and sleep problems in the general Norwegian working population, (2) to determine whether abusive supervision or the FKBP5 genotype is associated with these patterns, and (3) examine the interaction between abusive supervision and the FKBP5 genotype with regard to pain and sleep.

\section{Methods}

\section{Data collection}

The data was collected by Statistics Norway. A sample of 5,000 employees was randomly drawn from The Norwegian Central Employee Register. Inclusion criteria were 18-60 years of age and working at least $80 \%$ of full-time employment. The questionnaire was distributed by The Norwegian Postal Service in 2015. It was emphasized that data was anonymous and not reported to the employers. To those who consented, a saliva sample kit was distributed. The survey was approved by the Regional Committee for Medical Research for Eastern Norway.

\section{Instruments}

A 5-item version of Tepper's "Abusive supervision scale”, with response categories: "never”, "rarely”, "once in a while”, “quite often” and "very often or always" [2, 33]. Cronbach's alpha was 0.88 . The respondents were asked to indicate the intensity of various pain (headache, neck pain, upper back pain, low back pain and abdominal pain) and sleep (trouble falling asleep, trouble with coherent sleep and early awakening) complaints. With response categories: "not bothered", "a little bothered", "considerably bothered" and "seriously bothered".

\section{Genotyping}

As previously described [34] genomic DNA was extracted from saliva using OrageneRNA sample collection kit (DNA Genotech Inc. Kanata, Ontario, Canada). Briefly, single nucleotide polymorphism (SNP) genotyping was carried out using predesigned TaqMan SNP genotyping assays (Applied Biosystems, Foster City, CA, USA). In accordance with the procedure in earlier studies [34], an ABI 79000HT sequence detection system was used. Negative controls were included in every run. Approximately $10 \%$ of the samples were re-genotyped and the concordance rate was $100 \%$.

\section{Statistical analysis}

The average of the 5 items in the questionnaire was used to score abusive supervision. The pain and insomnia variables were dichotomised between "not bothered" and "a little bothered" to reflect the occurrence of complaints.

In the present study, the LCA was conducted in two steps. First, an unconditional LCA was performed to determine the appropriate number of latent classes for the pain and insomnia items, to identify meaningful phenotypes (subgroups) based on the individuals' responses [35]. To decide the optimal number of latent classes the Bayes Information Criterion (BIC) [36], Vuong-Lo-Mendall-Rubin (VLMR) likelihood ratio test and Lo-Mendell-Rubin (LMR) adjusted likelihood ratio test, were used [37].

Secondly, the LCA was extended by adding predictors, i.e., multinomial logistic regression analyses were conducted to examine whether abusive supervision and FKBP5 predicted membership of the latent classes. The final analysis was run as an LCA in one step, hence taking uncertainty of latent class membership into consideration.

Interactions (abusive supervision`FKBP5) and covariates (age and gender) were also tested. The analyses were run in four steps, first adding abusive supervision and FKBP5 in separate regressions to determine their main effects (step 1 and 2), then together to determine unique effects (step 3), then with interaction terms to determine any interaction effects (step 4). All steps were adjusted for age and gender. Missing data were handled by full information maximum likelihood (FIML). Statistical analyses were conducted using MPlus version 8.5, IBM SPSS Statistics 25 and STATA 16.

\section{Results}

In total, 1,608 (32\%) returned the questionnaire and 1,226 returned the saliva sample kit. 


\section{Sample characteristics}

A total of 1,073, i.e. 514 (48\%) males and 559 (52\%) females were successfully genotyped with regard to FKBP5 rs9470080 (see Table 1). The participants were classified based on which allele combination they had; homozygote CC, heterozygote CT or homozygote TT. Among the male cohort, 241 (46.8\%) were CC carriers, 226 (43.9\%) were CT carriers and $47(9.14 \%)$ were TT carriers. In the female cohort, 254 (45.4\%) were CC carriers, 253 (45.2\%) were CT carriers and $52(9.30 \%)$ were TT carriers. The average age was 44.5 for the CC variant, 45.7 for the CT variant and 46.1 for the TT variant. The mean level of experienced abusive supervision was $1.19(\mathrm{SD}=0.43)$ for the CC carriers, 1.18 $(\mathrm{SD}=0.43)$ for the $\mathrm{CT}$ carriers and $1.13(\mathrm{SD}=0.30)$ for the TT carriers. For the subjects with the CC genotype headache (59.5\%) and neck pain (59.3\%) were the most common complaints (see Table 1). For those with the CT genotype the most common complaints were lower back pain (57.4\%) and headache (56.5\%). Lastly, the most common complaints in those with the TT genotype were headache $(66.6 \%)$ and low back pain (60.6\%).

\section{Latent class enumeration}

The lowest BIC was observed for the four-class model, which also exhibited statistically significant VLMR and LMR, all of which point to this solution being preferable (see Supplementary Table 1). Hence, this solution was chosen. The classes exhibited distinct clinical patterns (Supplementary Table 2). Class 1 (23.3\%) exhibited high prevalence's of all complaints relative to the other classes (denoted "pain and insomnia"). Class 2 (24.5\%) exhibited low prevalence of pain complaints in combination with high prevalence of sleep problems ("insomnia"). Class 3 (26.3\%) exhibited higher pain prevalence's with low insomnia prevalence's and ("pain"). Lastly, class 4 (25.9\%) exhibited low prevalence's of all complaints ("few complaints”). The entropy for the final model with predictors, interactions and covariates was 0.741 . Figure 1 shows the patterns of the complaints in the final four classes.

\section{Latent class group predictors}

Multinomial logistic regressions, adjusted for age and gender were run to determine predictors of latent class membership. In these analyses (Table 2), the reference class was the "few complaints" class. In step 1 higher levels of abusive supervision were associated with higher odds of being in the insomnia class $(\mathrm{OR}=3.48,95 \% \mathrm{CI}=2.04,5.92)$, and the pain and insomnia class ( $\mathrm{OR}=2.20,95 \% \mathrm{CI}=1.12$, 4.30) compared to being in the "few complaints". Step 2 showed no statistically significant associations between classes and FKBP5. In Step 3, both predictors (abusive supervision and FKBP5) were included showing that it was more likely $(\mathrm{OR}=3.35,95 \% \mathrm{CI}=1.76,6.35)$ to be in class 1 , class $2(\mathrm{OR}=2.61,95 \% \mathrm{CI}=1.26,5.41)$ and class $3(\mathrm{OR}=2.47$, $95 \% \mathrm{CI}=1.41,5.34)$ compared to class 4 . A similar pattern was seen for Step 4, where the interaction between the two predictors was added, for class $1(\mathrm{OR}=5.05,95 \% \mathrm{CI}=1.60$, 15.8), class $2(\mathrm{OR}=4.15,95 \% \mathrm{CI}=1.25,13.7)$ and class 3 ( $\mathrm{OR}=3.86,95 \% \mathrm{CI}=1.06,14.0)$ for abusive supervision. No significant OR was found for FKBP5 nor the interaction effects.

Table 1: Characteristics of the sample by FKBP5 rs9470080; CC, CT and TT.

\begin{tabular}{|c|c|c|c|c|c|c|c|c|c|c|c|c|c|c|}
\hline & \multirow[t]{2}{*}{ Range } & \multicolumn{4}{|c|}{$\mathrm{CC}$} & \multicolumn{4}{|c|}{ CT } & \multicolumn{4}{|c|}{$T T$} & \multirow[t]{2}{*}{ Sum } \\
\hline & & $\mathbf{N}$ & $\%$ & Mean & SEM & $\mathbf{N}$ & $\%$ & Mean & SEM & $\mathbf{N}$ & $\%$ & Mean & SEM & \\
\hline Subjects & & & & & & & & & & & & & & 514 \\
\hline Males & & 241 & & & & 226 & & & & 47 & & & & 559 \\
\hline Females & & 254 & & & & 253 & & & & 52 & & & & 1,073 \\
\hline Age & & & & 44.5 & 0.44 & & & 45.7 & 0.44 & & & 46.1 & 1.00 & \\
\hline Abusive supervision & 1 to 5 & & & 1.19 & 0.01 & & & 1.18 & 0.02 & & & 1.13 & 0.03 & \\
\hline Headache & & 295 & 59.5 & & & 271 & 56.5 & & & 66 & $66.6 \mathrm{~g}$ & & & 632 \\
\hline Neck pain & & 294 & 59.3 & & & 263 & 54.9 & & & 53 & 53.5 & & & 610 \\
\hline Upper back pain & & 194 & 39.1 & & & 176 & 36.7 & & & 37 & 37.3 & & & 407 \\
\hline Low back pain & & 274 & 55.3 & & & 275 & 57.4 & & & 60 & 60.6 & & & 609 \\
\hline Abdominal pain & & 160 & 32.3 & & & 154 & 32.1 & & & 36 & 36.3 & & & 350 \\
\hline Trouble falling asleep & & 217 & 43.8 & & & 222 & 46.3 & & & 32 & 32.3 & & & 471 \\
\hline Trouble with coherent sleep & & 257 & 51.9 & & & 232 & 48.4 & & & 52 & 52.5 & & & 541 \\
\hline Early awakening & & 238 & 48.0 & & & 211 & 44.0 & & & 40 & 40.4 & & & 489 \\
\hline
\end{tabular}

$\mathrm{N}$ : number of subjects, SEM: standard error of mean. 


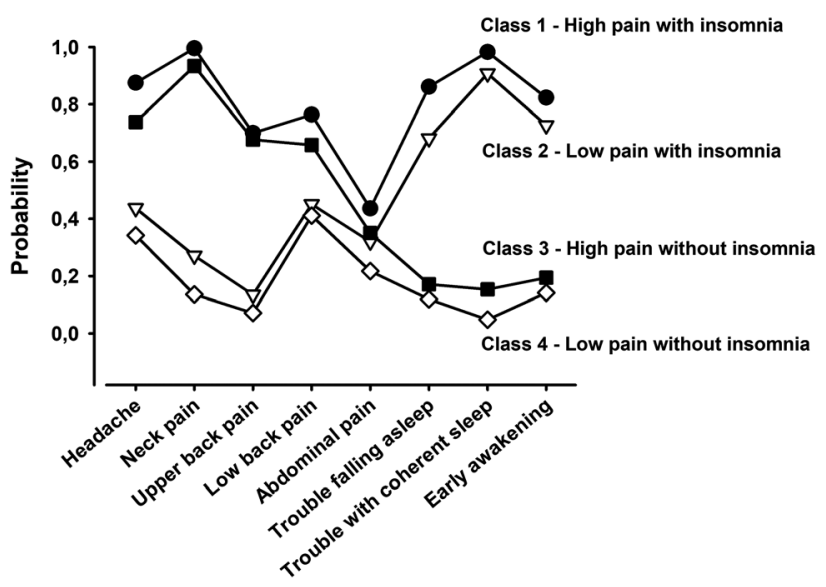

Figure 1: Visual representation of the four classes and the probability for each complaint (with predictors and covariates).

\section{Discussion}

This is the first study that examine the association between abusive supervision and latent classes based on response patterns [38] regarding health complaints in the general Norwegian working population. The testing of the model showed that the general working population could be grouped into four different classes. Clear class-differences regarding response patterns of the pain complaints and insomnia score were enlightened. Class 1 and 4 appeared to be opposites, with high probability of all complaints in class 1 and low probability of all complaints in class 4 . Likewise, class 2 and 3 also appeared to be opposites. Class 2 presented low pain with insomnia, whereas class 3 presented high pain without insomnia. These classes may reflect different clinical phenotypes, underscoring the utility of the LCA approach. Moreover, the analyses suggested that being subjected to abusive supervision influenced which pattern of complaints subjects experienced. This information may be of clinical importance as it provides a more complete understanding of complex conditions that may co-occur.

Interestingly, four different patterns, which also probably may be recognized in the clinic, were observed. Uncovering such subgroups may provide a more reliable prognosis and guide treatment of pain and insomnia patients, by tailoring the intervention based on the shared characteristics of the groups. However, we did not show any significant effects of FKBP5 genotype, indicating a rather low penetrance of this genetic variant. On the other hand, including the genetic factor increased the OR for abusive supervision indicating that it may influence the results. This could be due to the suppressor effect; adding one predictor increases the predictive power of another variable [39]. Still, whether or not abusive supervision promote pain and insomnia through FKBP5 mechanisms, remains unclear.

Several lines of evidence show that high quality sleep appears to improve the long-term prognosis of individuals

Table 2: Multinomial logistic regressions comparing different classes.

\begin{tabular}{|c|c|c|c|c|}
\hline & $\begin{array}{r}\text { High pain with insomnia } \\
\text { OR }(95 \% \mathrm{Cl})\end{array}$ & $\begin{array}{r}\text { Low pain with insomnia } \\
\text { OR }(95 \% \mathrm{Cl})\end{array}$ & $\begin{array}{r}\text { High pain without insomnia } \\
\text { OR }(95 \% \mathrm{Cl})\end{array}$ & $\begin{array}{l}\text { Low pain without insomnia } \\
\text { OR }(95 \% \mathrm{Cl})\end{array}$ \\
\hline Step 1 & & & & Reference \\
\hline Abusive supervision & $2.20(1.12,4.30)$ & $3.48(2.04,5.92)$ & $2.00(0.96,1.01)$ & \\
\hline \multicolumn{5}{|l|}{ Step 2} \\
\hline \multicolumn{5}{|l|}{ FKBP5 } \\
\hline CT & $1.01(0.58,1.75)$ & $0.93(0.56,1.53)$ & $0.73(0.39,1.37)$ & \\
\hline TT & $0.81(0.35,1.87)$ & $1.16(0.55,2.44)$ & $0.55(0.22,1.38)$ & \\
\hline \multicolumn{5}{|l|}{ Step 3} \\
\hline Abusive supervision & $3.35(1.76,6.35)$ & $2.61(1.26,5.41)$ & $2.47(1.41,5.34)$ & \\
\hline \multicolumn{5}{|l|}{ FKBP5 } \\
\hline CT & $0.72(0.46,1.14)$ & $0.99(0.55,1.77)$ & $0.92(0.57,1.49)$ & \\
\hline TT & $0.69(0.30,1.56)$ & $1.24(0.53,2.93)$ & $1.50(0.70,1.49)$ & \\
\hline \multicolumn{5}{|l|}{ Step 4} \\
\hline Abusive supervision & $5.05(1.60,15.8)$ & $4.15(1.25,13.7)$ & $3.86(1.06,14.0)$ & \\
\hline \multicolumn{5}{|l|}{ FKBP5 } \\
\hline CT & $1.89(0.40,8.99)$ & $2.68(0.51,14.0)$ & $2.51(0.43,14.4)$ & \\
\hline TT & $0.14(0.00,20.2)$ & $0.64(0.00,97.0)$ & $0.65(0.00,89.6)$ & \\
\hline Abusive supervision ${ }^{*} \mathrm{CT}$ & $0.42(0.11,1.60)$ & $0.40(0.10,1.61)$ & $0.40(0.09,1.77)$ & \\
\hline Abusive supervision ${ }^{*} \pi$ & $4.02(0.04,394)$ & $1.91(0.01,209)$ & $2.24(0.02,212)$ & \\
\hline
\end{tabular}

Statistically significant odds ratio are given in bold. All steps for all classes were adjusted for age and gender. FKBP5 reference is CC. OR; odds ratio, $\mathrm{Cl}$; confidence interval 
with tension-type headache, migraine and chronic musculoskeletal pain, for review see [26]. Moreover, it has been reported that poor quality of sleep or sleep restriction may be linked to pain hypersensitivity [25, 27]. However, given the present data, it seems reasonable to emphasize that this is not true for everyone. It is well documented that pain conditions such as chronic widespread pain, multisite pain and generalised pain are frequently observed in the general population [40, 41]. Earlier observations suggest that this could be explained by a phenomenon called hyperalgesic priming - which is a response to e.g. stressful events causing long-lasting changes in nociceptor function [42]. Based on this theory, it seems likely that stressful events, e.g., in the workplace, may lead to physiological changes that in turn could alter pain sensation. In addition, central sensitisation is thought to be highly important in chronicity and multisite pain complaints [42].

Moreover, pain complaints such as headache, musculoskeletal- and abdominal pain can all have multiple causes. Some may be caused by psychosocial challenges, others may have more explicit somatic origins, and often a combination of both is present. This is also true for problems relating to sleep and sleep quality. Based on the complexity and variety of the complaints investigated in this study it is tempting to emphasise the importance of considering a wider aspect of symptoms in clinical settings. The probability that different complaints impact each other makes it, even more, important to consider all complaints concurrently. Thus, by customising a treatment strategy on a group level, it seems probable that more advantageous results may be provided.

\section{Limitations}

Notably, the present study only showed associations, not cause-effect connections, between abusive supervision and the patterns of pain complaints and insomnia symptoms. Hence, a causal-effect relationship between these still needs further investigation. Moreover, even though the analysis showed four distinct classes, other subgroups with different (or more specific) symptom combinations, may also exist. Thus, inclusion of more detailed information on, for example, the insomnia symptoms (duration and frequency), type of headache and any potential structural origin of abdominal pain would also have been interesting.

Also, as the present study is based solely on self-reported information regarding both exposure and outcome, selfreport bias is a possibility [43]. Theoretically, those who report abusive supervision could also report more health problems based on personality and behaviour. However, the use of a psychometrically validated measure of abusive supervision, which asks respondents about specific occurrences of leadership behaviour rather than a global evaluation of the leader, should attenuate such bias. On the other hand, abusive supervision may be under-reported if subjects are afraid of repercussions. Therefore, all participants were informed that all information was collected anonymously. This reduces such bias [43].

Another potential restriction that needs to be mentioned is the accuracy of the pain sites reported. If the questionnaires were conducted with the support of a professional health care provider, the answers might have been different. Finally, the average response rate was $32 \%$, which seems to be in line with the current average [44]. There may be several reasons for a relatively low response rate. The participants were randomly selected at the individual level, which may have resulted in a lower response rate than what would have been achieved by employing a more active, organizational level recruitment strategy with clear benefits to the organization's work environment. Other possible reasons might be that the participants did not receive any compensation, financial or otherwise, or be comfortable donating genetic material. However, it is important to keep in mind that a low response rate is not in itself a cause of bias unless the nonresponse is selective with respect to variables of the study, and it seems unlikely to have affected associations of the present study [45].

\section{Conclusions}

The present study showed an association between abusive supervision and health complaints in the general working population where four pain- and insomnia response patterns were revealed. Awareness of the association between abusive supervision and these four pain and insomnia subgroups, and what separates them, may be of assistance in constructing prognosis and an optimal follow-up for those affected.

Acknowledgement: We thank Mina Eriksen for technical support in the lab.

Funding: The present work was supported by the Norwegian foundation: Et liv i bevegelse (ELIB).

Author contribution: ACS, JOC, DM, MBN and JG designed the research and analysed the data. ACS and JG wrote the manuscript with comments from JOC, DM and MBN.

Conflict of interests: The authors declare no competing financial or conflict of interest. 
Informed consent: Informed consent has been obtained from all individuals included in this study.

Ethical approval: The survey was approved by the Regional Committee for Medical Research for Eastern Norway(REK 2014/1725).

\section{References}

1. Fanavoll R, Nilsen TIL, Holtermann A, Mork PJ. Psychosocial work stress, leisure time physical exercise and the risk of chronic pain in the neck/shoulders: longitudinal data from the Norwegian HUNT Study. Int J Occup Med Environ Health 2016;29:585-95.

2. Tepper B. Consequences of abusive supervision. Acad Manag J 2000;43:178-90.

3. Tepper B, Duffy M, Hoobler J, Ensley M. Moderators of the relationships between coworkers' organizational citizenship behavior and fellow employees' attitudes. J Appl Psychol 2004; 89:455-65.

4. Herman JP, Ostrander MM, Mueller NK, Figueiredo H. Limbic system mechanisms of stress regulation: hypothalamo-pituitaryadrenocortical axis. Prog Neuro-Psychopharmacol Biol Psychiatry 2005;29:1201-13.

5. Smith SM, Vale WW. The role of the hypothalamic-pituitaryadrenal axis in neuroendocrine responses to stress. Dialogues Clin Neurosci 2006;8:383-95.

6. Russo SJ, Murrough JW, Han MH, Charney DS, Nestler EJ. Neurobiology of resilience. Nat Neurosci 2012;15:1475-84.

7. Vyas S, Rodrigues AJ, Silva JM, Tronche F, Almeida OF, Sousa N, et al. Chronic stress and glucocorticoids: from neuronal plasticity to neurodegeneration. Neural Plast 2016;2016:6391686.

8. Tsigos C, Kyrou I, Kassi E, Chrousos GP. Stress: endocrine physiology and pathophysiology, Feingold KR, Anawalt B, Boyce A, Chrousos G, de Herder WW, Dungan K, et al, editors. South Dartmouth (MA): Endotext, MDText.com, Inc.Copyright (C) 2000-2021, MDText.com, Inc.; 2000.

9. Sjöstedt E, Zhong W, Fagerberg L, Karlsson M, Mitsios N, Adori C, et al. An atlas of the protein-coding genes in the human, pig, and mouse brain. Science 2020;367: eaay5947.

10. Scammell JG, Denny WB, Valentine DL, Smith DF. Overexpression of the FK506-binding immunophilin FKBP51 is the common cause of glucocorticoid resistance in three New World primates. Gen Comp Endocrinol 2001;124:152-65.

11. Bortoluzzi A, Blaya C, da Rosa ED, Paim M, Rosa V, Leistner-Segal $S$, et al. What can HPA axis-linked genes tell us about anxiety disorders in adolescents? Trends Psychiatry Psychother 2015;37: 232-7.

12. Bryant RA, Felmingham KL, Liddell B, Das P, Malhi GS. Association of FKBP5 polymorphisms and resting-state activity in a frontotemporal-parietal network. Transl Psychiatry 2016;6: e925.

13. Khalid S, Tubbs RS. Neuroanatomy and neuropsychology of pain. Cureus 2017;9:e1754.

14. Willis WD, Westlund KN. Neuroanatomy of the pain system and of the pathways that modulate pain. J Clin Neurophysiol 1997;14: 2-31.

15. Morin LP. Neuroanatomy of the extended circadian rhythm system. Exp Neurol 2013;243:4-20.
16. Sannes AC, Christensen JO, Nielsen MB, Gjerstad J. The influence of age, gender and the FKBP5 genotype on subjective health complaints in the Norwegian working population. J Psychosom Res 2020;139:110264.

17. Kinge JM, Knudsen AK, Skirbekk V, Vollset SE. Musculoskeletal disorders in Norway: prevalence of chronicity and use of primary and specialist health care services. BMC Muscoskel Disord 2015; $16: 75$.

18. Ihlebaek C, Hansson TH, Laerum E, Brage S, Eriksen HR, Holm SH, et al. Prevalence of low back pain and sickness absence: a "borderline" study in Norway and Sweden. Scand J Publ Health 2006;34:555-8.

19. Aas RW, Tuntland H, Holte KA, Røe C, Lund T, Marklund S, et al. Workplace interventions for neck pain in workers. Cochrane Database Syst Rev 2011;2011:CD008160.

20. Stovner LJ, Andree C. Prevalence of headache in Europe: a review for the Eurolight project. J Headache Pain 2010;11:289-99.

21. Collaborators GBDH. Global, regional, and national burden of migraine and tension-type headache, 1990-2016: a systematic analysis for the global burden of disease study 2016. Lancet Neurol 2018;17:954-76.

22. Lyngberg AC, Rasmussen BK, Jørgensen T, Jensen R. Has the prevalence of migraine and tension-type headache changed over a 12-year period? A Danish population survey. Eur J Epidemiol 2005;20:243-9.

23. Fredriksen TA, Antonaci F, Sjaastad O. Cervicogenic headache: too important to be left un-diagnosed. J Headache Pain 2015;16:6.

24. Nilsson $\mathrm{N}$. The prevalence of cervicogenic headache in a random population sample of 20-59 year olds. Spine 1995;20:1884-8.

25. Alsaadi SM, McAuley JH, Hush JM, Lo S, Lin CW, Williams CM, et al. Poor sleep quality is strongly associated with subsequent pain intensity in patients with acute low back pain. Arthritis Rheum 2014;66:1388-94.

26. Finan PH, Goodin BR, Smith MT. The association of sleep and pain: an update and a path forward. J Pain 2013;14:1539-52.

27. Bollinger T, Schibler U. Circadian rhythms - from genes to physiology and disease. Swiss Med Wkly 2014;144:w13984.

28. Schwartz MD, Kilduff TS. The neurobiology of sleep and wakefulness. Psychiatr Clin 2015;38:615-44.

29. Lim J, Dinges DF. A meta-analysis of the impact of short-term sleep deprivation on cognitive variables. Psychol Bull 2010;136: 375-89.

30. Matre D, Hu L, Viken LA, Hjelle IB, Wigemyr M, Knardahl S, et al. Experimental sleep restriction facilitates pain and electrically induced cortical responses. Sleep 2015;38:1607-17.

31. Hammerschlag AR, Stringer S, de Leeuw CA, Sniekers S, Taskesen $E$, Watanabe K, et al. Genome-wide association analysis of insomnia complaints identifies risk genes and genetic overlap with psychiatric and metabolic traits. Nat Genet 2017;49: 1584-92.

32. Roth T. Insomnia: definition, prevalence, etiology, and consequences. J Clin Sleep Med 2007;3:S7-10.

33. Hamid RA, Juhdi N, Ismail M, Abdullah NA, Hamid A. Abusive supervision and workplace deviance as moderated by spiritual intelligence: an empirical study of Selangor employees. Malays J Soc Space 2016;12:191-202.

34. Jacobsen DP, Nielsen MB, Einarsen S, Gjerstad J. Negative social acts and pain: evidence of a workplace bullying and 5-HTT genotype interaction. Scand J Work Environ Health 2018;44:283-90. 
35. Meisingset I, Vasseljen O, Vøllestad NK, Robinson HS, Woodhouse A, Engebretsen KB, et al. Novel approach towards musculoskeletal phenotypes. Eur J Pain 2020;24: 921-32.

36. Dziak JJ, Coffman DL, Lanza ST, Li R, Jermiin LS. Sensitivity and specificity of information criteria. Brief Bioinform 2020;21: 553-65.

37. Nylund-Gibson K, Asparouhov T, Muthén B. Deciding on the number of classes in latent class analysis and growth mixture modeling: a Monte Carlo simulation study. Struct Equ Modeling 2007;14:535-69.

38. Kongsted A, Nielsen AM. Latent class analysis in health research. J Physiother 2017;63:55-8.

39. Watson D, Clark LA, Chmielewski M, Kotov R. The value of suppressor effects in explicating the construct validity of symptom measures. Psychol Assess 2013;25:929-41.

40. Christensen JO, Nielsen MB, Finne LB, Knardahl S.

Comprehensive profiles of psychological and social work factors as predictors of site-specific and multi-site pain. Scand J Work Environ Health 2018;44:291-302.
41. Mansfield KE, Sim J, Jordan JL, Jordan KP. A systematic review and meta-analysis of the prevalence of chronic widespread pain in the general population. Pain 2016;157:55-64.

42. Ji R-R, Nackley A, Huh Y, Terrando N, Maixner W. Neuroinflammation and central sensitization in chronic and widespread pain. Anesthesiology 2018;129:343-66.

43. Podsakoff $P$, MacKenzie S, Lee J-Y, Podsakoff N. Common method biases in behavioral research: a critical review of the literature and recommended remedies. J Appl Psychol 2003;88:879-903.

44. Stedman R, Connelly N, Heberlein T, Allred S. The end of the (research) world as we know it? Understanding and coping with declining response rates to mail surveys. Soc Nat Resour 2019;32:1139-54.

45. Schalm RL, Kelloway EK. The relationship between response rate and effect size in occupational health psychology research. J Occup Health Psychol 2001;6:160-3.

Supplementary Material: The online version of this article offers supplementary material (https://doi.org/10.1515/sjpain-2021-0124). 